\title{
Proposta de otimização da produtividade do setor de coleta externa de um hemocentro do estado de São Paulo
}

Productivity optimization proposal in external collection sector in a state of São Paulo's blood center

\section{Renê Felippe Ohtani ${ }^{1}$, Jaqueline Oliveira Valdeviño Nascimento ${ }^{2}$, Ticiane Nishimoto $^{3}$, Susana Lambert ${ }^{4}$, Nancy Val y Val Peres da Mota ${ }^{5}$}

1. Médico. Residente em medicina preventiva do Programa de Estudos Avançados em Administração Hospitalar e de Sistemas de Saúde (PROAHSA) do Hospital das Clínicas da Faculdade de Medicina da USP (HCFMUSP), São Paulo, SP.

2. Enfermeira. Aprimoranda do PROAHSA HCFMUSP, São Paulo, SP.

3. Gerontóloga. Aprimoranda do PROAHSA HCFMUSP, São Paulo, SP.

4. Diretora de Relações Externas e Intercâmbios da Fundação Pró-Sangue Hemocentro de São Paulo; São Paulo, SP.

5. Médica. Membro do corpo técnico do PROAHSA HCFMUSP, São Paulo, SP.

\section{RESUMO}

Introdução: Segundo dados brasileiros coletados em 2015, nota-se uma queda do número de coleta de bolsas de sangue em comparação com o ano anterior. Um grande hemocentro do estado de São Paulo oferece um serviço de coleta externa, enviando uma equipe para coletar bolsas no local de uma instituição que deseja realizar uma campanha de doação de sangue. Com o conhecimento deste serviço, foi gerada uma demanda superior à oferta oferecida pelo hemocentro. Objetivo: O objetivo deste artigo é descrever os processos do serviço de coleta externa, realizar uma análise dos resultados do ano de 2017 em comparação com os demais postos de coleta deste hemocentro e propor estratégias de melhoria sobre esta atividade. Material e métodos: Foram realizadas entrevistas, análise de documentos e dados dos resultados operacionais. A partir desta análise, foi proposto quatro cenários de 
melhoria sobre a operação. Resultados: A coleta externa ocorre apenas em dois dias da semana e tem seu fluxo similar ao adotado nos demais postos fixos. A maior diferença é em relação ao agendamento, vistoria do local, armazenamento das bolsas e transporte de equipamentos, materiais e funcionários. Dados indicam que a produtividade da coleta externa foi de apenas 3.367 bolsas, sendo a segunda área com menor produção. Quando ajustado aos seus dias de funcionamento, a coleta externa apresenta a segunda maior produtividade entre todos os postos. A relação entre bolsas coletadas e capacidade operacional de todos os postos mostra uma utilização da infraestrutura inferior a $50 \%$ da capacidade máxima, sendo a coleta externa o setor que tem melhores valores sobre este indicador. Com a identificação desta oportunidade, foram propostos 4 cenários que permitiriam aumentar 0 número de bolsas coletadas. Discussão: A baixa porcentagem de utilização da capacidade operacional da maioria dos postos pode ser explicada pela inadequação do quadro de funcionários da organização. Por representar um serviço com demanda programada desde o início do ano, a coleta externa tem pontos fortes e oportunidades de melhoria para aumentar o número de bolsas coletadas. Dessa forma, uma Matriz B.A.S.I.C.O. foi montada a partir dos cenários propostos nos resultados. Conclusão: Com o entendimento do processo realizado durante uma coleta externa de bolsas de sangue, foi possível identificar o principal fator limitante da operação através da análise dos dados de produção. Destaca-se também o impacto dessas coletas sobre o resultado total do hemocentro, bem como sua capacidade operacional e a relação entre os demais postos. Ao final das análises, observou-se a existência de um grande potencial de melhoria sobre o setor de coletas externas. Dessa forma os quatro cenários de melhoria podem proporcionar um melhor resultado com baixo investimento adicional, podendo ser priorizado o cenário 1, 0 primeiro ranqueado da Matriz B.A.S.I.C.O.

Palavras-Chaves: banco de sangue; gestão em saúde; doadores de sangue.

\section{ABSTRACT}

Introduction: According to Brazilian data collected in 2015, there is a reduction in the blood bag collection compared to the previous year. A large blood center of the State of São Paulo offers an external collection service, sending a team to collect blood bags in the area of the institution that wishes to start a blood donation campaign. Knowing this service, a large demand was created in face of this activity's offer. Objective: The objective of this article is to describe the processes of the external collection service, realize a result analysis of the year 2017 in comparison with all the collection outposts of this blood center and propose improvement strategies about this operation. Methods and Materials: Interviews, document assessment and data analysis of the operational results were made. From these actions, four improvement scenes about the operation were proposed. Results: The external collection service occurs during two weekdays and has it's processes' flux is similar as the one adopted by the other outposts. The biggest difference is in the scheduling, site inspection, storing and 
transporting of the blood bags, materials and workers. The data indicates that the external collection's productivity was of 3.367 blood bags, being the second to last production area. When adapted to its working days, the external collection presents itself as the second largest productivity between all the areas. The blood bag collection numbers and operational capacity ratio of all outposts shows a structural utilization lower than $50 \%$ of its maximum capacity, with the external collection displaying the best result of this indicator. By identifying this opportunity, 4 scenarios were proposed that would allow a growth in the number of collected blood bags. Discussion: The low percentage of operational capacity utilization of most of the outposts can be explained by the inadequacy of the organization's staff. Because of the service's known demand since the beginning of the year, the external collection has great strengths and opportunities to increase the number of collected blood bags. By so, a B.A.S.I.C.O. Matrix was devised from the proposed scenarios in the Result section. Conclusion: The understanding of the process allowed a identification of the main limiting factor of this operation through the data analysis realized. It was also stood out the impact of this type of collection on the overall blood center's result, as well its operational capacity and relationship with other organization's outposts. By the end of the analysis, it was observed the existence of a great improvement potential on the external collection sector. With the four optimization scenarios, it is possible to achieve a better result with low additional investment, with the scenario 1 being the first that can be prioritized according to the B.A.S.I.C.O. matrix results.

Keywords: blood banks; health management; blood donors.

\section{INTRODUÇÃO}

A transfusão sanguínea é um procedimento médico indicado para o tratamento de algumas doenças específicas, além de determinados casos de emergências. Tanto o uso do próprio sangue quanto o uso de hemocomponentes derivados de seu processamento, é possível obter melhora da condição de clínica de alguns pacientes ${ }^{(1)}$. A obtenção do sangue para realização dessas terapias ocorre em diversas instituições que se dedicam exclusivamente à coleta e/ou transfusão de sangue através da captação de doadores voluntários.

Apesar de ser uma terapia indispensável na área da saúde, ainda há pouca conscientização de sua importância entre a população do Brasil. Em comparação com o ano anterior, em 2015 foi registrado um crescimento na demanda por bolsas de sangue, considerando o aumento de 91.717 transfusões sanguíneas ${ }^{(2)}$. Em contrapartida, no mesmo período observou-se uma queda de 28.102 coletas de bolsas de sangue, acompanhado de um aumento de 734.926 doadores inaptos (condições não favoráveis para doação de sangue segundo triagem clínica) ${ }^{(2)}$. 
Tentando evitar um cenário de oferta insuficiente para a demanda deste produto e entendendo as dificuldades do doador inserido neste contexto, um grande hemocentro de São Paulo criou em 1995 um serviço de coleta externa, que se encontra em atividade nos dias atuais, para complementar a produção de suas unidades fixas. Mediante um agendamento prévio, qualquer organização que manifeste interesse em realizar campanhas de doação de sangue pode receber uma equipe de coleta de bolsas de sangue in loco às terças e quintas-feiras, após avaliação de especialista para averiguar a adequação das instalações. Assim, há um benefício para os doadores, evitando o deslocamento de um grande número de pessoas por parte da organização, e a possibilidade de aumentar o número de bolsas coletadas por parte do hemocentro, atualmente representando $4 \%$ do número total de bolsas coletadas no ano.

Esta iniciativa se mostrou tão valiosa a ponto de gerar uma demanda muito superior aos dois períodos semanais ofertados, resultado evidenciado pela reserva de todos os horários disponíveis em 2018 em apenas 15 dias após abertura da agenda.

\section{OBJETIVO}

O objetivo deste artigo é descrever os processos do serviço de coleta externa realizada pelo hemocentro em questão, realizar uma análise dos resultados da coleta externa no ano de 2017, comparar os resultados entre os postos de coleta deste hemocentro e propor estratégias de melhoria sobre esta atividade.

\section{MATERIAL E MÉTODOS}

Foram realizadas entrevistas com perguntas estruturadas de resposta aberta para coletar dados sobre o fluxo do processo de coleta externa, tempo de funcionamento da atividade, composição do quadro de funcionários, período e forma de seu agendamento e quantidade de bolsas coletadas. Também foram feitas perguntas sobre o horário de funcionamento de todos os postos fixos de coleta de sangue, quadro de funcionário destes postos e quantidade de bolsas coletadas no ano de 2017.

Os entrevistados foram selecionados pelo tempo de experiência com a coleta externa. No período da entrevista, ocupavam os seguintes cargos na organização: médico responsável pelo setor de coletas externas, diretoria de enfermagem, coordenadoria de educação permanente e diretoria de tecnologia e informação.

O setor de Tecnologia e Informação da instituição disponibilizou dados provenientes do sistema "Ciclo do Sangue" referentes ao número de bolsas de sangue coletadas na coleta externa e em cada posto de coleta durante 0 período de janeiro a setembro de 2017. Os dados continham informações sobre número de bolsas coletadas por período em todas as datas de 
funcionamento de cada unidade coleta. A partir dessas informações, foram criadas tabelas e gráficos para comparar a produção de cada posto em relação aos dias da semana e meses do ano.

Para que fosse possível comparar a real relação das coletas externas com os demais postos elaborou-se a Tabela 2, que elenca o número de bolsas coletadas por mês em cada posto fixo de coleta, considerando apenas os dias e período de funcionamento das coletas externas.

Através dos dados obtidos, foi possível identificar a capacidade operacional máxima dos pontos de doação de sangue. A fórmula extraída do Planeja Sangue ${ }^{(3)}$ permite identificar o número máximo de bolsas de sangue que uma unidade consegue coletar durante um mês. $O$ cálculo descrito abaixo leva em consideração o número de cadeiras disponíveis para coleta, número de horas por dia e o número de dias de funcionamento da unidade.

Capacidade operacional mensal $=3$ coletas por hora $x$ número de horas de funcionamento do serviço por dia x número de cadeiras disponíveis no local de coleta x número de dias de funcionamento do serviço no mês

O resultado da fórmula e os dados de produção possibilitaram a criação de um indicador composto pela relação entre o número total bolsas coletadas em cada mês e a capacidade operacional no mesmo mês. Por conta de variação no horário de funcionamento e disponibilidade de equipamentos dos postos, foi necessário calcular a capacidade operacional máxima de cada mês para encontrar a média geral no período. Esse indicador foi utilizado para demonstrar o percentual uso da capacidade operacional máxima mensal de cada unidade de coleta de sangue.

Ao final, foram descritos quatro cenários a partir das oportunidades de melhoria encontrados após esta análise de dados. A seguir, os cenários foram aplicados à uma ferramenta chamada Matriz B.A.S.I.C.O.

Este instrumento auxilia a priorização de cenários após a atribuição de uma pontuação entre 1 a 5 quando considerados os seguintes critérios: benefícios da organização; abrangência da solução; satisfação dos funcionários; investimentos necessários; impacto sobre os clientes; capacidade de operacionalização. A somatória da pontuação de cada cenário permite a criação de um ranking de priorização das ações propostas.

\section{RESULTADOS}

Descrição dos processos da coleta externa 
Para que exista o serviço de coleta externa, três são as áreas envolvidas: o hemocentro, o principal posto de doação de sangue deste hemocentro e uma empresa terceirizada.

O processo se inicia com a abertura da agenda da coleta externa e a solicitação de inscrição por parte da organização externa voluntária, através de contato via telefone ou internet. Em uma situação de indisponibilidade de vagas, a instituição solicitante é cadastrada em uma lista de espera, podendo ser contatada para agendamento "de urgência" no caso de abertura de alguma vaga.

Com a confirmação do agendamento, uma visita às instalações da organização demandante é realizada em até um mês de antecedência, para avaliar o local disposto para a realização da coleta externa quanto à capacidade da infraestrutura e existência de possíveis riscos que possam prejudicar a coleta, além da distância e tempo de trajeto até o local de processamento das bolsas.

Se o ambiente for declarado apto a receber a equipe do hemocentro, é criada uma escala dos membros que formarão a equipe: 1 médico, 2 entrevistadores (biomédico ou enfermeiro), 4 técnicos de enfermagem e 2 motoristas. São separados também todo material e equipamentos necessários para efetuar a coleta, sendo dispostos: 8 macas, 8 aparelhos para coleta, 80 bolsas précadastradas, recipiente de descarte de material biológico, caixa térmica para armazenamento das bolsas e lanche para os candidatos.

Fluxo e processo da coleta externa é similar ao do posto de coleta: o doador é recepcionado e é feito a realização do cadastro, caso ainda não tenha. É então realizada uma fase de triagem para avaliação de aptidão física, e em seguida, uma entrevista que tem como objetivo entender o perfil do doador. Antes de realizar a doação, todos os doadores realizam o voto de autoexclusão*. É então realizada a coleta, com duração média de 20 minutos, e o doador recebe um lanche para que se sinta melhor no caso de reação adversa à doação.

* Voto de autoexclusão: etapa exigida por lei durante a doação de sangue. É neste momento que o doador consente que seu sangue pode ser usado para fins terapêuticos e autoriza o uso da bolsa gerada a partir da coleta. O voto é feito de forma privativa.

A realização da coleta na instituição tem limite máximo de 4 horas de duração, e ocorre às terças e quintas-feiras, podendo ser realizada com exclusividade em outros períodos mediante um agendamento prévio. Por conta de limitação de equipamento de armazenamento, foi estabelecido um limite de 70 bolsas coletadas para cada dia de visitas externas. Caso este limite seja atingido, o cadastro é interrompido, sendo retomado apenas se houver a eliminação de algum candidato ou bolsas e se ainda existirem voluntários para doação. As bolsas que são coletadas retornam para o posto principal do hemocentro, onde serão processadas da mesma forma que as bolsas coletadas nos outros postos.

\section{Coletas de bolsas de sangue}


Na Tabela 1 observa-se que o número total de bolsas coletadas pelo hemocentro foi de 89.149. O posto $\mathrm{E}$, o maior em relação a espaço físico, horário de funcionamento, número de funcionários e equipamentos assistenciais, foi o posto que apresentou maior produção, totalizando 49.282 bolsas. O estabelecimento que teve menor produtividade foi o posto $\mathbf{C}$, que apresentou uma coleta de 6.543 bolsas de sangue. A coleta externa foi a área de operação que menos coletou bolsas no período. No total, foram 3.367 bolsas coletadas externamente aos postos fixos, apresentando um pico máximo de 624 coletas no mês de junho.

Tabela 1. Total de bolsas coletadas em cada posto nos meses do ano. São Paulo, 2017.

\begin{tabular}{|l|r|r|r|r|r|r|r|}
\hline $\begin{array}{l}\text { Mês de } \\
\mathbf{2 0 1 7}\end{array}$ & \multicolumn{1}{l|l}{$\begin{array}{l}\text { Posto } \\
\text { A }\end{array}$} & $\begin{array}{l}\text { Posto } \\
\text { B }\end{array}$ & $\begin{array}{l}\text { Posto } \\
\text { C }\end{array}$ & $\begin{array}{l}\text { Posto } \\
\text { D }\end{array}$ & \multicolumn{1}{l|l}{$\begin{array}{l}\text { Posto } \\
\text { E }\end{array}$} & $\begin{array}{l}\text { Coleta } \\
\text { externa }\end{array}$ & \multicolumn{1}{l|}{ Total } \\
\hline Janeiro & 834 & 1.270 & 754 & 728 & 5.210 & 0 & 8.796 \\
\hline Fevereiro & 726 & 1.550 & 683 & 636 & 5.334 & 208 & 9.137 \\
\hline Março & 1.024 & 1.635 & 748 & 825 & 5.484 & 462 & 10.178 \\
\hline Abril & 749 & 1.378 & 543 & 727 & 4.971 & 399 & 8.767 \\
\hline Maio & 1.028 & 1.759 & 989 & 1.000 & 5.781 & 438 & 10.995 \\
\hline Junho & 1.151 & 1.788 & 850 & 1.036 & 6.056 & 624 & 11.505 \\
\hline Julho & 879 & 1.565 & 699 & 1.058 & 5.472 & 190 & 9.863 \\
\hline Agosto & 1.069 & 1.696 & 744 & 278 & 5.446 & 456 & 9.689 \\
\hline Setembro & 913 & 1.660 & 533 & 995 & 5.528 & 590 & 10.219 \\
\hline Total & $\mathbf{8 . 3 7 3}$ & $\mathbf{1 4 . 3 0 1}$ & $\mathbf{6 . 5 4 3}$ & $\mathbf{7 . 2 8 3}$ & $\mathbf{4 9 . 2 8 2}$ & $\mathbf{3 . 3 6 7}$ & $\mathbf{8 9 . 1 4 9}$ \\
\hline
\end{tabular}

Com a adaptação que permitiu a criação da Tabela 2, observa-se que o serviço de coleta externa é a atividade que apresenta a segunda maior produtividade da organização, ficando atrás apenas do posto E. Nota-se também que a diferença no número de coleta de bolsas entre a coleta externa e o posto $\mathbf{B}$, serviço com terceira maior produtividade, é de mais de mil bolsas. Destaca-se também que o mês no qual a coleta externa apresentou maior produtividade foi em março. Excluindo-se o mês de janeiro, período em que o serviço não apresenta atividade, o mês com menor produção foi julho, fato que difere em relação aos demais postos.

Tabela 2. Total de bolsas coletadas em cada posto às terças e quintas-feiras nos meses do ano. São Paulo, 2017.

\begin{tabular}{|l|l|l|l|l|l|l|}
\hline Mês de 2017 & $\begin{array}{l}\text { Posto } \\
\text { A }\end{array}$ & $\begin{array}{l}\text { Posto } \\
\text { B }\end{array}$ & $\begin{array}{l}\text { Posto } \\
\text { C }\end{array}$ & $\begin{array}{l}\text { Posto } \\
\text { D }\end{array}$ & $\begin{array}{l}\text { Posto } \\
\text { E }\end{array}$ & $\begin{array}{l}\text { Coleta } \\
\text { externa }\end{array}$ \\
\hline Janeiro & 158 & 187 & 105 & 68 & 575 & 0 \\
\hline
\end{tabular}




\begin{tabular}{|l|r|r|r|r|r|r|}
\hline Fevereiro & 143 & 168 & 77 & 0 & 573 & 208 \\
\hline Março & 178 & 215 & 109 & 14 & 603 & 462 \\
\hline Abril & 157 & 195 & 121 & 101 & 566 & 399 \\
\hline Maio & 197 & 215 & 133 & 129 & 618 & 438 \\
\hline Junho & 215 & 183 & 115 & 115 & 688 & 338 \\
\hline Julho & 290 & 152 & 175 & 121 & 563 & 190 \\
\hline Agosto & 255 & 230 & 201 & 184 & 689 & 456 \\
\hline Setembro & 137 & 186 & 152 & 112 & 642 & 361 \\
\hline Total & $\mathbf{1 7 3 0}$ & $\mathbf{1 7 3 1}$ & $\mathbf{1 1 8 8}$ & $\mathbf{8 4 4}$ & $\mathbf{5 5 1 7}$ & $\mathbf{2 8 5 2}$ \\
\hline
\end{tabular}

\section{Capacidade Operacional}

Na Tabela 3 observa-se que a capacidade operacional de 22.088 do posto E é a maior entre os serviços desta instituição, destacando-se pela grande diferença todos os outros postos de coleta. A coleta externa apresentou capacidade operacional de 816, a menor entre os serviços de coleta deste hemocentro.

Apesar de nenhum posto conseguir atingir metade de sua capacidade operacional máxima, nota-se que, em contrapartida, o serviço que apresentou melhor uso de sua capacidade operacional, com $45,85 \%$ do seu potencial foi a coleta externa, e o posto $\mathbf{C}$ o serviço que menos utilizou seu potencial de coleta, apresentando apenas $22,16 \%$ do uso de sua capacidade operacional.

Tabela 3. Média de bolsas coletadas por mês, média da capacidade operacional máxima mensal e relação entre a média de bolsas coletadas por mês e capacidade operacional máxima mensal em cada unidade de coleta. São Paulo, 2017.

\begin{tabular}{|l|l|l|l|}
\hline $\begin{array}{l}\text { Posto de } \\
\text { coleta }\end{array}$ & $\begin{array}{l}\text { Média de bolsas } \\
\text { coletadas por } \\
\text { mês }\end{array}$ & $\begin{array}{l}\text { Média da capacidade } \\
\text { operacional máxima } \\
\text { mensal }\end{array}$ & $\begin{array}{l}\text { Relação entre média de } \\
\text { bolsas coletadas por mês } \\
\text { e capacidade operacional } \\
\text { máxima mensal }\end{array}$ \\
\hline Posto A & 930 & 2312 & $40,24 \%$ \\
\hline Posto B & 1589 & 6810 & $23,33 \%$ \\
\hline Posto C & 727 & 3280 & $22,16 \%$ \\
\hline Posto D & 917 & 3314 & $27,69 \%$ \\
\hline Posto E & 5475 & 22088 & $24,79 \%$ \\
\hline Coleta & 374 & 816 & $45,85 \%$ \\
\hline
\end{tabular}




\section{DISCUSSÃO}

\section{Descrição dos processos da coleta externa}

Com a necessidade de um bom mapeamento dos processos e uma logística bem estruturada, as coletas externas exigem uma qualificação e treinamento constante dos funcionários para garantir a segurança dos processos de coleta. A partir do processo descrito, nota-se que a etapa que mais restringe o uso da capacidade operacional máxima é o armazenamento e o transporte das bolsas coletadas.

A existência de desafios logísticos para preservação do material coletado e transporte de funcionários, equipamentos e materiais impedem que mais de 70 bolsas sejam coletadas, dentro das condições em que a operação é realizada atualmente. Esta etapa é uma das mais importantes do processo, e esse estudo identificou-a como uma grande oportunidade para aumentar a produção do setor, com a vantagem de não adicionar custos com mais funcionários, treinamento ou equipamentos.

\section{Coleta de bolsas de sangue}

A limitação dos períodos reservados para a coleta externa faz com que seus resultados sejam os menores entre os demais postos analisados. A observação dos números totais de produção no período estudado não representa um resultado verdadeiro da operação, e por isso foi realizado uma comparação adequando os dados obtidos de todos os postos aos dias em que a coleta externa é realizada. Esta adaptação permitiu visualizar que o setor da coleta externa é o segundo maior produtor entre todos os postos, ficando atrás apenas do posto central, que tem maior visibilidade dos doadores e da mídia, além de melhor localização geográfica, melhor acesso aos doadores e melhor preparo técnico dos funcionários. Os demais postos possuem menor estrutura física e de equipamentos, e historicamente têm menor movimento de doadores, consequentemente produção muito menor que o posto central (posto E) e a coleta externa.

\section{Capacidade operacional}

Poucos são os dados disponíveis de outros hemocentros, e principalmente sobre capacidade operacional. Com grande visibilidade e relevância, posto E possui o maior número de equipamentos para coleta das bolsas de sangue e maior tempo de funcionamento, visando melhorar sua operação durante os finais de semana e feriados, dias em que o posto tem maior movimento. A 
queda importante em sua busca nos demais dias da semana, conforme o Gráfico 1, faz com que este posto tenha uma menor produção e uma ociosidade no uso de seus equipamentos, diminuindo seu aproveitamento em relação à capacidade operacional.

Gráfico 1. Quantidade de bolsas de sangue coletadas em cada dia da semana no posto E. São Paulo, 2017.

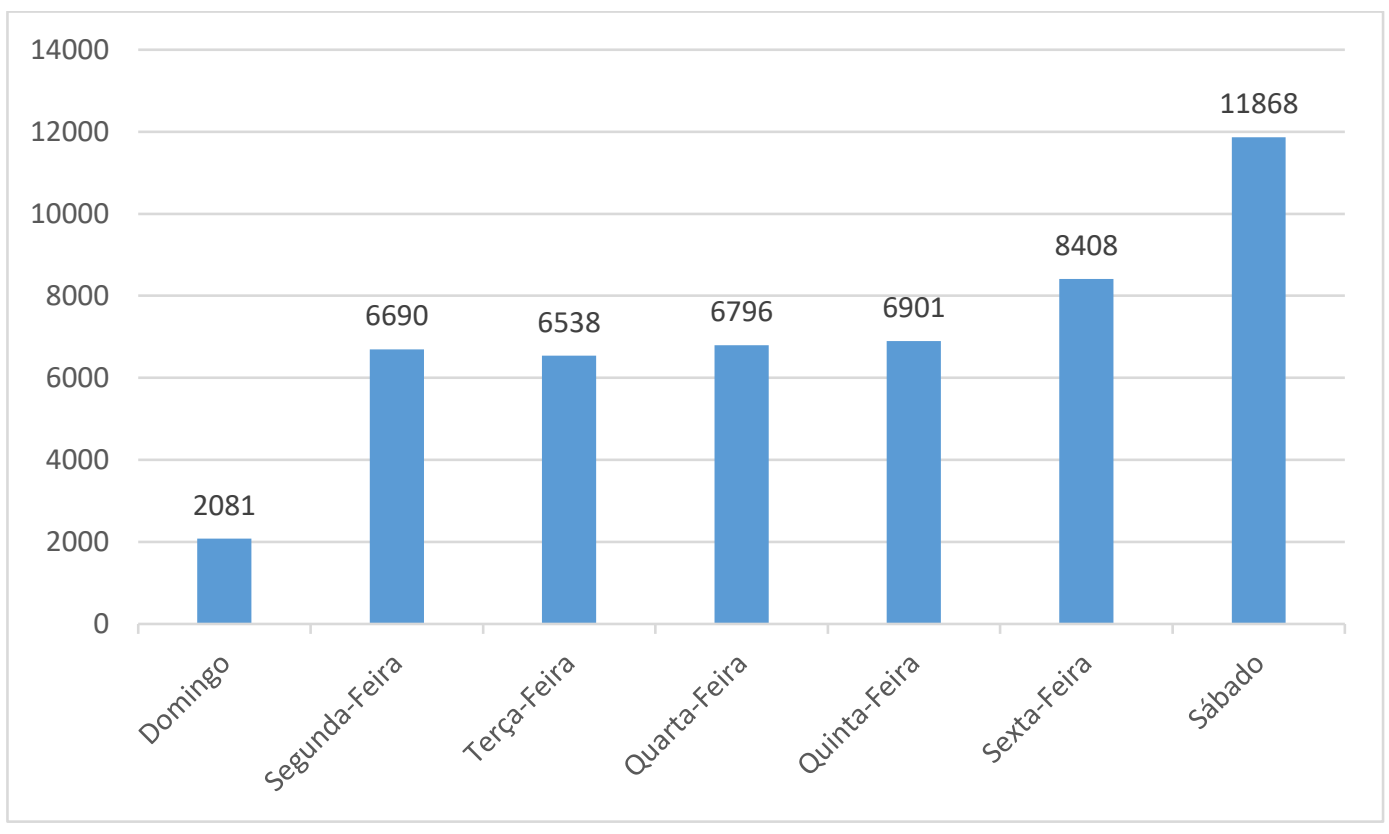

Iniciativas de contratação de novos funcionários da área assistencial não aconteceram durante 0 ano, representando fator que pode prejudicar o alcance ótimo da capacidade operacional por causar grande impacto sobre a força de trabalho de todos os postos.

Dessa forma, com um número adequado de funcionários para os equipamentos dispostos à coleta, as coletas externas são o setor desta instituição que apresentam melhores resultados em relação à sua capacidade operacional. Como já descrito, o fator que mais impacta em sua limitação é o armazenamento e transporte das bolsas.

No detalhamento mensal expresso pelo Gráfico 1, é possível notar que apenas no mês de setembro a relação entre bolsas coletadas e a capacidade operacional do mês foi superior a $50 \%$. Nota-se uma queda da coleta e da capacidade operacional no mês de julho, fato que pode ser explicado por conta do período de férias em universidade e algumas empresas.

Porém, a característica de maior destaque das coletas externas está na previsibilidade da demanda. Com o agendamento de todas as coletas externas a serem realizadas no ano, a instituição consegue estimar um número de bolsas que este setor irá produzir, diferentemente dos demais postos que são 
dependentes de demanda espontânea dose seus doadores. Essa informação permite a instituição se planejar e adequar todos os seus recursos à esta atividade, otimizando sua capacidade operacional para cada coleta e reduzindo a ociosidade de produção ao máximo.

\section{Propostas de otimização de resultados sobre a coleta externa}

Identificando essa oportunidade envolvendo o setor de coletas externas, foram propostos quatro cenários que permitiriam este hemocentro aumentar o número de coleta de bolsas de sangue:

\section{Cenário 1 - Atuação com a capacidade operacional ideal: Foi identificada a existência de um gargalo no armazenamento e transporte das bolsas de sangue. Essa restrição impõe um limite de 70 bolsas por coleta externa, número que fica abaixo da capacidade operacional máxima de 120 bolsas calculada para esta atividade. Esta oportunidade é uma alternativa para aperfeiçoar a coleta de bolsas de sangue deste serviço que possui demanda conhecida realizando apenas alterações sobre as etapas logísticas do processo, mantendo a atividade principal inalterada.}

Cenário 2 - Coletas externas aos sábados: Ao se criar a possibilidade da realização de coletas externas aos sábados, pouco irá se alterar em relação à dinâmica de trabalho dos demais postos, gerando poucos impactos produtivos nos postos fixos e aumentando o número de bolsas coletadas. A partir do momento em que um hemocentro, que necessita de doações voluntárias de sangue, nega o desejo manifestado por diversas empresas e instituições em receber a equipe de coleta externa dentro de sua estrutura física sem custos diretos, cria-se uma situação paradoxal para sua imagem. Para que seja possível absorver essa demanda superior à oferta de serviços, sem alterar os processos de execução da coleta externa, seria vantajosa a existência de mais períodos nos quais a coleta externa pudesse ser realizada.

Cenário 3 - Aumento dos períodos de coleta externa: Com o mesmo objetivo do cenário anterior, sugere-se a expansão dos horários disponíveis para realização de coleta externa do hemocentro. Sabendo que, normalmente, a coleta externa ocorre às terças e quintas-feiras pela manhã, a realização deste serviço para outras instituições no período da tarde destes mesmos dias poderia causar um baixo impacto na operação, porém traria um bom resultado produtivo.

\section{Cenário 4 - Coletas externas nos dias de menor movimento dos postos fixos: Por conta do baixo número de produção de bolsas de sangue de algumas unidades fixas de coleta, em comparação com outras unidades e inclusive com a coleta externa, conclui-se a existência de uma ociosidade da capacidade operacional. Devido a essa situação, o fechamento do posto com menor coleta de bolsas por um dia da semana e o direcionamento de seus funcionários e equipamentos para}


uma instituição próxima que esteja na lista de espera da coleta externa pode ser vantajoso pelo possível ganho na produtividade se comparado a um dia típico desta unidade. Por apresentar um maior planejamento e reorganização dos processos assistenciais, é necessário que seja avaliado o custo de oportunidade para a realização deste cenário e quais os benefícios previstos.

Matriz B.A.S.I.C.O.

A aplicação dos cenários nos critérios da Matriz B.A.S.I.C.O. resultou em um ranking representado pela Tabela 4 .

Tabela 4. Matriz B.A.S.I.C.O.

\begin{tabular}{|c|c|c|c|c|}
\hline & Cenário 1 & Cenário 2 & Cenário 3 & Cenário 4 \\
\hline $\begin{array}{l}\text { Benefícios para a } \\
\text { Organização }\end{array}$ & $\begin{array}{l}\text { Atuação com } \\
\text { a capacidade } \\
\text { operacional } \\
\text { ideal }\end{array}$ & $\begin{array}{l}\text { Expansão do } \\
\text { número de } \\
\text { coletas } \\
\text { realizadas, sem } \\
\text { interferir na } \\
\text { dinâmica de } \\
\text { funcionamento } \\
\text { dos demais } \\
\text { postos de coleta }\end{array}$ & $\begin{array}{l}\text { Expansão do } \\
\text { número de coletas } \\
\text { realizadas }\end{array}$ & $\begin{array}{l}\text { Expansão do } \\
\text { número de coletas } \\
\text { realizadas através } \\
\text { da ampliação de um } \\
\text { dia de coleta } \\
\text { externa nos dias } \\
\text { com menor } \\
\text { movimentação dos } \\
\text { demais postos de } \\
\text { coleta }\end{array}$ \\
\hline $\begin{array}{l}\text { Abrangência da } \\
\text { Solução }\end{array}$ & $\begin{array}{l}\text { Atuação com } \\
\text { a capacidade } \\
\text { operacional }\end{array}$ & $\begin{array}{l}\text { Aumento do } \\
\text { número de } \\
\text { instituições } \\
\text { participantes da } \\
\text { coleta externa }\end{array}$ & $\begin{array}{l}\text { Aumento do } \\
\text { número de coletas, } \\
\text { mas sem atingir a } \\
\text { capacidade } \\
\text { operacional }\end{array}$ & $\begin{array}{l}\text { Aumento do } \\
\text { número de coletas, } \\
\text { sem atingir a } \\
\text { capacidade } \\
\text { operacional }\end{array}$ \\
\hline $\begin{array}{l}\text { Satisfação dos } \\
\text { Funcionários }\end{array}$ & $\begin{array}{l}\text { Quantidade } \\
\text { de horas } \\
\text { trabalhadas } \\
\text { sem alteração }\end{array}$ & $\begin{array}{l}\text { Revezamento } \\
\text { entre todos os } \\
\text { profissionais } \\
\text { dos postos de } \\
\text { coleta para os } \\
\text { plantões de final } \\
\text { de semana }\end{array}$ & $\begin{array}{l}\text { Dimensionamento } \\
\text { de } \mathrm{RH} \text { (escala única } \\
\text { de todos os postos } \\
\text { de coleta) }\end{array}$ & $\begin{array}{l}\text { Dimensionamento } \\
\text { de RH (escala única } \\
\text { de todos os postos } \\
\text { de coleta) }\end{array}$ \\
\hline $\begin{array}{l}\text { Investimentos } \\
\text { Necessários }\end{array}$ & $\begin{array}{l}\text { Reajuste no } \\
\text { número de } \\
\text { transportes } \\
\text { utilizados }\end{array}$ & $\begin{array}{l}\text { Divulgação do } \\
\text { novo período } \\
\text { disponibilizado } \\
\text { para a } \\
\text { realização de } \\
\text { coleta externa }\end{array}$ & $\begin{array}{l}\text { Profissionais } \\
\text { escalados já fazem } \\
\text { parte do quadro de } \\
\text { funcionários da } \\
\text { Fundação Pró- } \\
\text { Sangue }\end{array}$ & $\begin{array}{l}\text { Mobilização dos } \\
\text { profissionais para a } \\
\text { realização de mais } \\
\text { coletas externas }\end{array}$ \\
\hline $\begin{array}{l}\text { Impacto sobre os } \\
\text { Clientes }\end{array}$ & $\begin{array}{l}\text { Possibilidade } \\
\text { de maior } n^{\circ} \\
\text { de doadores }\end{array}$ & $\begin{array}{l}\text { Melhora na } \\
\text { oferta das } \\
\text { coletas externas } \\
\text { (redução da fila } \\
\text { de espera) }\end{array}$ & $\begin{array}{l}\text { Possibilidade de } \\
\text { maior } n^{\circ} \text { de } \\
\text { doadores }\end{array}$ & $\begin{array}{l}\text { Possibilidade de } \\
\text { maior } n^{\circ} \text { de } \\
\text { doadores }\end{array}$ \\
\hline Capacidade de & Planejamento & Escala unificada & Reorganização no & Escala unificada de \\
\hline
\end{tabular}




\begin{tabular}{|l|l|l|l|l|}
\hline Operacionalização & $\begin{array}{l}\text { orçamentário } \\
\text { anual (curto } \\
\text { prazo) }\end{array}$ & $\begin{array}{l}\text { de todos os } \\
\text { postos de coleta } \\
\text { (médio a longo } \\
\text { prazo) }\end{array}$ & $\begin{array}{l}\text { agendamento dos } \\
\text { veículos (médio a } \\
\text { longo prazo) }\end{array}$ & $\begin{array}{l}\text { todos os postos de } \\
\text { coleta e } \\
\text { reorganização no } \\
\text { agendamento dos } \\
\text { veículos (médio a } \\
\text { longo prazo) }\end{array}$ \\
\hline Total & 25 & 24 & 23 & 22 \\
\hline Ranking & 10 & 20 & $3^{\circ}$ & $4^{\circ}$ \\
\hline
\end{tabular}

\section{CONCLUSÃO}

Com o entendimento do processo realizado durante uma coleta externa de bolsas de sangue, foi possível identificar o principal fator limitante da operação através da análise dos dados de produção. Destaca-se também o impacto dessas coletas sobre o resultado total do hemocentro, bem como sua capacidade operacional e a relação entre os demais postos.

Ao final das análises, observou-se a existência de um grande potencial de melhoria sobre o setor de coletas externas. Dessa forma os quatro cenários de melhoria podem proporcionar um melhor resultado com baixo investimento adicional, podendo ser priorizado o cenário 1, o primeiro ranqueado da Matriz B.A.S.I.C.O.

\section{REFERÊNCIAS}

1. Yaddanapudi, Sandhya et al. Indications for blood and blood product transfusion. Indian Journal of Anaesthesia. Chandigarh, India. Volume 58, 2014.

2. Caderno de informação - sangue e hemoderivados: dados de 2015. Brasília: Ministério da Saúde; 2017. 15 p.

3. Souza, Mariluce Karla Bomfim de et al (orgs). PlanejaSangue. Cursos em Planejamento e Gestão de Sistema e Serviços de Saúde. Guia para Estudos/Mariluce Karla Bomfim de Souza et al... Salvador/Brasília, 2 ed e ver., 2016. 
Recebido: 23 de março de 2018. Publicado: 20 de abril de 2018

Correspondência: Renê Felippe Ohtani. E-mail: reneohtani@gmail.com

Conflito de interesses: os autores declararam não haver conflito de interesses.

(C) This is an Open Access article distributed under the terms of the Creative Commons Attribution License, which permits unrestricted use, distribution, and reproduction in any medium, provided the original work is properly cited 